\title{
Inactivation of the Nuclear Orphan Receptor COUP-TFII by Small Chemicals
}

Rémy Le Guével ${ }^{1,2,3^{*}}$, Frédérik Oger ${ }^{4,5,6,7{ }^{*}}$, Celia P. Martinez-Jimenez ${ }^{8}$, Maud Bizot ${ }^{1,2}$, Céline Gheeraert $^{4,5,6,7}$, François Firmin ${ }^{4,5,6,7}$, Maheul Ploton ${ }^{4,5,6,7}$, Miroslava Kretova ${ }^{9}$, Gaëlle Palierne ${ }^{1,2}$, Bart Staels ${ }^{4,5,6,7}$, Peter Barath $^{9}$, lannis Talianidis ${ }^{8}$, Philippe Lefebvre ${ }^{4,5,6,7}$, Jérôme Eeckhoute ${ }^{4,5,6,7}$, Gilles Salbert ${ }^{1,2}$

${ }^{1}$ Université Rennes 1, F-35042, Rennes, France

${ }^{2}$ UMR6290 CNRS, Team SP@RTE, Campus de Beaulieu, F-35042, Rennes, France

${ }^{3}$ SFR Biosit, UMS 3480 CNRS, US 018 INSERM, Campus de Villejean, F-35043, France

${ }^{4}$ European Genomic Institute for Diabetes (EGID), FR 3508, F-59000 Lille, France

${ }^{5}$ Inserm UMR U1011, F-59000, Lille, France

${ }^{6}$ Université Lille 2, F-59000, Lille, France

${ }^{7}$ Institut Pasteur de Lille, F-59019, Lille, France

${ }^{8}$ B.S.R.C. "Alexander Fleming", 34 Fleming Street, 16672, Vari, Greece

${ }^{9}$ Cancer Research Institute BMC, Slovak Academy of Sciences, Dúbravská cesta 9, SK-845 05 Bratislava, Slovak Republic

* These two authors contributed equally to this study.

Contact:

Pr. Gilles Salbert

CNRS UMR6290-Université Rennes 1

Team SP@RTE-Campus de Beaulieu

35042, Rennes, France

Phone: +33223236625

Fax : +33223236794

E-mail: gilles.salbert@univ-rennes1.fr

Running title: Chemical inactivation of COUP-TFII 
Key words: nuclear receptor, COUP-TF, Cyp7a1, methoxynaphthol

\begin{abstract}
Chicken ovalbumin upstream promoter-transcription factor $\|$ (COUP-TFII/NR2F2) is an orphan member of the nuclear receptor family of transcription factors whose activities are modulated upon binding of small molecules into an hydrophobic ligand binding pocket (LBP). Although the LBP of COUP-TFII is filled with aromatic amino-acid side chains, alternative modes of ligand binding could potentially lead to regulation of the orphan receptor. Here, we screened a synthetic and natural compound library in a yeast one-hybrid assay and identified 4-methoxynaphthol as an inhibitor of COUP-TFII. This synthetic inhibitor was able to counteract processes either positively or negatively regulated by COUP-TFII in different mammalian cell systems. Hence, we demonstrate that the true orphan receptor COUP-TFII can be targeted by small chemicals which could be used to study the physiological functions of COUP-TFII or to counteract detrimental COUP-TFII activities in various pathological conditions.
\end{abstract}

\title{
Introduction
}

COUP-TFI (NR2F1) and COUP-TFII (NR2F2) belong to the nuclear receptor (NR) family which is made up of 48 members in human. ${ }^{1}$ COUP-TFI/II regulate developmental processes and control important functions during adult life. ${ }^{2-7}$ COUP-TFs were first designated as " orphan " nuclear receptors in view of their homology with founding members of the family and their lack of known ligand. X-ray determination of the structure of the COUP-TFII ligand binding domain (LBD) revealed that it is probably a true orphan, due to an autorepressed conformation in which helix a 10/11 is bent into the putative hydrophobic ligand-binding pocket (PDB 3cjw). ${ }^{8}$ True orphans are uncommon proteins in the family since among the 41 crystallized vertebrate receptors (see Suppl. Table I), only 7 of them (i.e. COUP-TFII, Nurr1, Nur77, PNR, TR4, REV-ERB $\alpha$ and REV-ERB $\beta$ ) show a ligand binding cavity which is fully occupied by bulky amino-acid side-chains. ${ }^{8-10}$ Surprisingly, despite the absence of a classical ligand binding cavity in the crystal structure of COUP-TFII, Kruse et al. ${ }^{8}$ showed that, under certain circumstances, COUP-TFII could be activated by retinoic acid and interpreted this observation as an indication that a ligand binding pocket might form in some cellular contexts, leading to an active conformation in which helix a $10 / 11$ is straightened. ${ }^{8}$ NR ligand binding cavities have highly different volumes ranging from $100 \AA^{3}$ (ERR1) ${ }^{11}$ to $1600 \AA^{3}$ (SF-1, ${ }^{12}$ see Suppl. Table I). The hydrophobic 
cavity found in the modeled putative active conformation of COUP-TFII LBD with a straight helix a10/11 was predicted to have a volume of $600-700 \AA^{3}$ and could thus easily accommodate small ligands such as retinoic acid. ${ }^{8}$ Alternatively, activation of COUP-TFII by retinoic acid could result from binding to a cryptic site within the COUP-TFII LBD. Interestingly, Nurr1 can be activated by small chemicals that bind to hydrophobic grooves at the surface of the ligand-binding domain, ${ }^{13,14}$ suggesting that even true orphans can be drug targets. On the other hand, small LBP may accommodate large ligands, inducing conformational disturbances which promote alternative activation states. ${ }^{15}$ In opposition to the more classic transcriptional activation or repression model, conditioned by coactivator or corepressor recruitment, these alternative activation states may induce nuclear degradation and cytoplasmic or nuclear aggregation of the targeted NR, as demonstrated for the estrogen receptor alpha bound by antagonists. ${ }^{16,17}$ In the present report, we describe the discovery of small molecules regulating COUP-TFII and their use for the functional inspection of the COUP-TFII orphan receptor in adipocyte differentiation and liver gene expression.

\section{Results and Discussion}

Identification of a « surface » binding pocket within the hCOUP-TFII LBD.

Despite the absence of a buried ligand binding pocket, the published structure of hCOUP-TFII LBD (PDB 3cjw) was interrogated for the presence of interaction surfaces by various on-line softwares. 3cjw was first screened for putative ligand binding sites with Q-SiteFinder. ${ }^{18}$ Two sites, out of 10 predicted (see supplementary Fig. S1), displayed a volume of $271 \AA^{3}$ (site 1) and a volume of $254 \AA^{3}$ (site 2), potentially able to accommodate small molecules. This first analysis was followed by a search for protein pockets and cavities with Pocket-Finder, a software implementing LIGSITE. ${ }^{19}$ PocketFinder predicted that site 1 is associated with a cavity of $208 \AA^{3}$, whereas no significant cavity was predicted at site 2 (Suppl. Fig. S1). Lastly, applying PPI-Pred, a software designed to predict proteinprotein interaction surfaces,$^{20}$ predicted that site 2 is integrated into a large protein-protein interaction interface, whereas site 1 only partially overlaps with a protein-protein interaction domain (Suppl. Fig. S1). Hence, site 1 is likely to provide a docking site for a putative non-proteinaceous ligand. According to the above analysis, this molecule would dock into a cavity (hereafter called " surface pocket ») ranging from 208 to $271 \AA^{3}$ and delineated by residues from helices $\alpha 3, \alpha 7$ and $\alpha 10 / 11$ (Fig. 1A, 1B and $1 \mathrm{C}$ ). Residues lining the cavity are mostly hydrophobic, although a few polar aminoacids may 
provide hydrogen bonds to putative ligands (C213, R385, H291). Nonetheless, it should be stressed that residues L269 to V285 between helices $\alpha 5$ and $\alpha 7$ are missing in the solved structure. ${ }^{8}$ Depending on their respective spatial positioning, these residues could eventually reduce the size of the detected cavity. However, superimposition of COUP-TFII, RXR $\alpha$ and HNF $4 \alpha$ LBD structures indicated that the missing residues in COUP-TFII LBD structure are not likely to fill the cavity since the corresponding space is mostly occupied by residues forming the start of $\alpha 3$ in RXR $\alpha$ and HNF4 $\alpha$, residues which are positioned away from the cavity in COUP-TFII, a situation which occurs also in the structure of other autorepressed LBDs like those of PNR and TR4 (Suppl. Fig. S2).

We next mutated residues either found in the putative surface pocket or being buried in the filled classical pocket (Fig. 1D) and analyzed the residual activity of the mutants in a yeast one-hybrid system (Fig. 1E and 1F). In such a system, wild-type (wt) COUP-TFII was able to induce transcription of the reporter gene. The activity of mutants W249A, F253A and F295A, was severely compromised. Side chains of residues W249, F253 and F295 fill the classical pocket ${ }^{8}$ and hence are likely to be required for proper folding of the protein. Interestingly, mutants for residues found in the surface cavity (I212A, C213A, F288A and H291A) maintained high transcriptional activation, indicating that these three aminoacids do not play a major role in folding the LBD.

\section{COUP-TFII is inactivated by naphthol derivatives.}

The shape, size and nature of the predicted surface cavity in COUP-TFII LBD suggested that it could accommodate small natural or synthetic ligands composed of functionalized phenyl rings. We thus designed a focused library of 67 commercially available compounds, most of which met the above mentioned criteria (see Suppl. Table II). This library was screened in the yeast one-hybrid system measuring wtCOUP-TFIl mediated transcriptional activity (Fig. 2A). No compound was able to increase transcription of the reporter gene. Conversely, 4-methoxynaphtalen-1-ol, 6methoxynaphtalen-2-ol, 2-naphtol and, to a lesser 1,3-dihydroxynaphtalen (compounds 62, 64, 61 and 46 respectively Suppl. Table II) inhibited wtCOUP-TFII activity (Fig. 2A). Interestingly, these four compounds were structurally related to naphthol (Fig. 2B) and the most active molecule (4methoxynaphthalene-1-ol, thereafter called 4-MNol) had an IC50 of $100 \mu \mathrm{M}$ in the yeast one hybrid assay. Interestingly, the calculated volume of 4-MNol was about $180 \AA^{3}$, thus below the estimated volume of the surface pocket of COUP-TFII $\left(273 \AA^{3}\right)$. Binding of small compounds to nuclear receptor 
surfaces has already been shown to occur in small hydrophobic grooves implicated in cofactor recruitment by the estrogen receptor $\alpha$ and Nurr1. ${ }^{13,14,21}$ However, the COUP-TFII surface cavity where 4-MNol docks is away from the known cofactor interaction site for NRs (opposite end of helix a11) which is occupied by helix $\alpha 12$ in COUP-TFII LBD structure. ${ }^{8}$ Hence, 4-MNol inhibition of COUPTFIl might not rely on a direct blockade of cofactor interaction, but could rather stabilize an autorepressed conformation. Docking simulations showed that 4-MNol could bind in the predicted pocket with the methyl group fitting deeply into the cavity and contacting F382, one of the residues involved in filling the classical ligand-binding pocket (Fig. 2C and 2D). Interestingly, the oxygen atom of the hydroxy group of 4-MNol could potentially form hydrogen bonds with C213 and R385 (Fig. 2D). Since the SH group of $\mathrm{C} 213$ is engaged in hydrogen bonding with F382 (oxygen atom of the peptide bond between F382 and F383 at the end of $\alpha 10 / 11$ ) in the COUP-TFII LBD crystal, ${ }^{8}$ binding of 4-MNol to C213 is likely to destabilize this intramolecular bond, an event which could indirectly impact on the positioning of $\alpha 12$. According to this hypothesis, 4-MNol was tested in yeast one-hybrid assay using COUP-TFII mutant C213A. Data showed a reduced sensitivity to the inhibitory action of the compound in the context of the mutated receptor (Fig. 2E), suggesting that 4-MNol can bind within the predicted cavity, an event which could lead to structural alterations reducing the transcriptional activity of COUPTFII. To probe such structural modifications, in vitro-translated full-length wtCOUP-TFII was incubated with increasing amounts of chymotrypsin in a limited proteolysis assay. Short fragments resulting from the partial cleavage of the full-length protein were more amenable to degradation by chymotrypsin in the presence of 4-MNol (Fig. 2F). Since these fragments are likely to result from the cleavage of the ligand binding domain as described for nuclear receptors, ${ }^{22}$ these results indicate that 4-MNol might destabilize COUP-TFII LBD. Consequences of a direct interaction between 4-MNol and COUP-TFII were next investigated by gel shift experiments. Translation of the nuclear receptor in the presence of 4-MNol decreased wtCOUP-TFII binding to DNA while it did not affect the rate of in vitro synthesis (Fig. 3A and 3B). This was probably not due to a direct effect of $4 \mathrm{MNol}$ on the DNA binding domain of COUP-TFII since COUP-TFII transcriptional activity was inhibited in yeast one-hybrid assay in which COUP-TFII binds DNA through the LexA-DBD moiety. When 4-MNol was added after protein synthesis, during incubation with the DNA probe, the decrease in DNA binding was less pronounced (Fig. 3C). When dithiothreitol (DTT) was added in the gel-shift assay, DNA binding of COUP-TFII, in vitro translated in the presence of $4-\mathrm{MNol}$, was partially restored. These data, together with the 
predicted interaction of 4-MNol with $\mathrm{C} 213$, indicate that the redox-state of COUP-TFII could be altered by 4-MNol. This observation point to a prominent role of sulfhydryl groups in the functionality of COUP-TFII. Hence, mutant C213A was tested in gel shift assay for its susceptibility to 4-MNol. In these assays, binding of C213A COUP-TFII to DNA was found to be less affected than binding of wtCOUP-TFII (Fig. 3D and Fig. S3). These data support the 3D docking model suggesting that 4-MNol anchoring to the receptor LBD is likely to rely, at least partly, on hydrogen bonding between C213 and the oxygen of the hydroxy group of 4-MNol. Nonetheless, it has been demonstrated that 4-MNol can undergo oxidation into Russig's blue. ${ }^{23}$ Such a reaction would be slowed down by DTT in vitro, suggesting that active form of 4-MNol inhibiting COUP-TFII activity in our assays could actually be oxidized 4-MNol. We thus analyzed a putative interaction of Russig's blue with the surface pocket of COUP-TFII LBD through docking experiments. Data revealed that Russig's blue could fit in the pocket mainly through hydrophobic interaction and anchoring to C213 and R385 (Fig. S4A). Binding energies indicated that Russig's blue would have a higher affinity than 4-MNol for this site (Fig. S4B). Moreover, these docking experiments predicted that one phenyl ring of Russig's blue would protrude from COUP-TFII LBD hence possibly favoring aggregation and degradation. Collectively, these data favor a model in which 4-MNol induces a conformational switch of COUP-TFII LBD leading to aggregation and/or degradation of the protein.

\section{4-MNol decreases COUP-TFII protein levels and activity in hepatoma cells.}

COUP-TFII is highly expressed in liver and kidney where it has been proposed to regulate a number of metabolic pathways. ${ }^{24}$ Co-transfection experiments have been used previously to investigate the regulatory functions of COUP-TFs (COUP-TFI and COUP-TFII) on putative target genes. It has been for instance established in vitro that COUP-TFs down-regulate genes such as Apoliprotein $A 1, A I V, B$ and $\mathrm{CIII},{ }^{25-27}$ whereas COUP-TFII is positively involved in the HNF-4 $\alpha$-dependent Cyp7a1 gene activation. ${ }^{28-30}$ In this context, we analysed the expression of COUP-TFII target genes by RT-qPCR in human liver hepatoma cells (HepG2) treated with 4-MNol. The 4-MNol toxicity was found to be limited even at $100 \mu \mathrm{M}$ (Fig. 4A) and RT-qPCR data revealed that Cyp7a1 mRNA levels gradually decreased with increasing 4-MNol concentrations whereas ApoA1 mRNA levels remained unchanged up to 50 $\mu \mathrm{M}$ 4-MNol (Fig. 4B). In order to analyse whether the effects of 4-MNol on Cyp7a1 gene expression were dependent on COUP-TFII, we next transfected HepG2 cells with siRNAs targeting COUP-TFII. In 
these conditions, 4-MNol showed a reduced ability to inhibit Cyp7a1 expression (Fig. 4C), indicating that its effects are at least partly conveyed through inhibition of the orphan receptor. COUP-TFII is known to co-activate HNF-4a on the Cyp7a1 gene ${ }^{28}$ and its presence at the Cyp7a1 promoter in liver cells requires HNF-4a (Talianidis, unpublished data). To evaluate the impact of 4-MNol on molecular events occurring at the Cyp7a1 promoter, we then run chromatin immunoprecipitation (ChIP) assays. The results demonstrated that COUP-TFII binding to the promoter of Cyp7a1 was reduced by 4-MNol (Fig. 4D). Interestingly, the reduction in Cyp7a1 promoter occupancy by COUP-TFII did not alter the engagement of HNF-4a but was detrimental to the recruitment of activated RNA Pol II and the histone acetyl-transferase CBP (Fig. 4D). Accordingly, histone H3 acetylation at the Cyp7a1 promoter was lower in 4-MNol-treated cells (Fig. 4D). In accordance with a lower engagement of COUP-TFII at the Cyp7a1 promoter upon 4-MNol treatment, COUP-TFII protein levels were dramatically reduced by 4MNol (Fig. 4E), despite a slightly increased COUP-TFII mRNA expression (Fig. 4C). In order to gain insights into the mechanisms leading to reduced COUP-TFII protein levels in HepG2 cells treated with 4-MNol, we first inhibited the proteasome by adding MG132 to HepG2 cells. Surprisingly, MG132 reduced COUP-TFII levels, and combining MG132 with 4-MNol led to further reduction in COUP-TFII (Fig. 4E). Hence, it is unlikely that 4-MNol induces degradation of COUP-TFII through the ubiquitin/proteasome pathway. Alternatively, aggregates of COUP-TFII might form upon 4-MNol binding and be degraded through autophagy. We then hypothesized that if such aggregates are induced by 4-MNol, they could be evidenced in the form of foci by immunofluorescence experiments. Results indicated that HepG2 cells treated with 4-MNol tend to have an increased number of fluorescent foci, specially in the cytosol (Fig. 4F), suggesting that indeed 4-MNol induces aggregation of COUP-TFII in HepG2 cells.

\section{4-MNol enhances 3T3-L1 adipocyte differentiation.}

Since 4-MNol reduced COUP-TFII activity in HepG2 cells, we hypothesized that 4-MNol could also affect adipocyte differentiation, a process inhibited by COUP-TFII, partly through binding to and repression of the PPAR $\gamma$ gene. ${ }^{7,31,32}$ Hence, 3T3-L1 cells (a fibroblastic cell line able to differentiate into mature adipocytes) were treated with 4-MNol following the protocol displayed in Fig. 5A. Negative control was obtained from confluent cells cultured with regular medium without any stimuli (Fig. 5A, control -), whereas positive control corresponded to differentiation induced by dexamethasone and 
insulin (Fig. 5A, control +). Adipocyte differentiation was then evaluated by Oil Red O (ORO) staining of triglycerides (TGs) and direct TG content determination 8 days after dexamethasone addition. As observed for HepG2 cells, the viability of 3T3-L1 cells was not affected by 4-MNol up to $100 \mu \mathrm{M}$ (Fig. 5B). As expected, dexamethasone alone induced partial adipocyte differentiation (Fig. 5C) and when 4-MNol and dexamethasone were combined, TG positive cells and TG content increased in a dosedependent manner (Fig. 5D,E). These data indicate that 4-MNol enhanced dexamethasone-induced conversion of 3T3-L1 fibroblasts to adipocytes. To further analyze the effects of 4-MNol on in vitro adipocyte differentiation, mRNA expression levels of two main adipogenic markers (PPARY and Adiponectin) were determined by RT-qPCR. Cells were treated with increasing concentrations of 4MNol and PPARY and adiponectin mRNA expression was monitored 2, 4, 6 and 8 days after induction of differentiation. In accordance with the observed increase in the percentage of adipocytes in 4-MNol treated cells, expression of both PPARY and adiponectin mRNAs was enhanced in a time and dosedependent manner by $4-\mathrm{MNol}$ (Fig. 5F). Taken together, these data demonstrate that 4-MNol enhanced adipocyte differentiation as well as mRNA expression of adipogenic markers such as PPARY and adiponectin Despite 4-MNol reduced COUP-TFII protein levels in HepG2 cells, a similar effect of 4-MNol was not evidenced in 3T3-L1 (data not shown). This observation points to diverse molecular outputs of 4-MNol/COUP-TFII interaction in different cell types. Our EMSA analysis suggests that 4-MNol oxidation could generate more efficient inhibitors of COUP-TFII which could lead to protein aggregation in cells. Accordingly, 4-MNol could be oxidized in cells expressing cytochrome P450 enzymes like HepG2 cells. ${ }^{23}$ Hence, several 4-MNol-derived molecular species might be at the origin of a seemingly differential behaviour of the COUP-TFII protein in different cell types.

\section{Conclusion}

We report here that activity of the orphan nuclear receptor COUP-TFII can be modulated by small chemicals belonging to the naphthol family. Challenging COUP-TFII-regulated processes with 4-MNol allowed us to conclude that this compound can efficiently target COUP-TFII not only in the yeast onehybrid system but also in different mammalian cell types. Hence, 4-MNol can be used as a new tool to probe the role of COUP-TFII in various biological processes. Importantly, these data further reveal that COUP-TFII could be considered as a therapeutic target since the activity of this orphan nuclear receptor can be manipulated through the action of small chemicals. 


\section{Experimental procedures}

\section{Materials and cloning}

All yeast media were from Sigma-Aldrich. The synthetic compound library was built from individual compounds from Sigma-Aldrich. For screening of compound activity we used the DupLex-A ${ }^{T M}$ yeast two-hybrid system from OriGene Technologies, Inc. (USA). Full-length human HNF-4a1 and human COUP-TFII aa52-414 were fused in frame with the LexA DNA binding domain (DBD) in the one-hybrid vector pEG202 (OriGene Technologies, Inc.) as already described. ${ }^{33}$ All COUP-TFII mutant plasmids were prepared with the QuickChangeTM kit (Stratagene, USA) as described by the manufacturer with oligonucleotides from Proligo (France - see Suppl. information). All mutations were confirmed by sequencing.

\section{One hybrid assays and library screening}

Compounds from the targeted library were tested in a 96 well-plate format assay as described,$^{34}$ using the LexADBD-COUP-TFII one-hybrid system in which the production of $\beta$-galactosidase in yeast cells reflects the transcriptional activity of the fusion protein. Cells were treated with compounds solubilized in DMSO (1\% final concentration). Control experiments run with DMSO alone were used to correct for the solvent effect. After cell lysis, $\beta$-galactosidase activity was revealed with the fluorescent substrate 4-methylumbelliferyl $\beta$-D-galactopyranoside (4MUG, Sigma-Aldrich) at a final concentration of 40 $\mu \mathrm{g} / \mathrm{mL}$.

\section{Electromobility shift assay}

Electromobility shift assay were performed in $10 \mu \mathrm{l}$ reaction consisting of $20 \mathrm{mM}$ Hepes $(\mathrm{pH} 7.9), 350$ $\mathrm{mM} \mathrm{KCl}, 25 \mathrm{mM} \mathrm{MgCl}_{2}, 25 \%$ glycerol, $1 \mathrm{mM}$ dithiothreitol, $100 \mu \mathrm{M} \mathrm{ZnCl}_{2}$. In vitro translated COUP TFII was added to the binding buffer, followed by $2 \mu \mathrm{g}$ poly ( $\mathrm{dl}-\mathrm{dC}$ ) and $10000 \mathrm{cpm}$ of ${ }^{32} \mathrm{P}$-labeled double stranded DR1 probe (5'-TGCGAGGTCAAAGGTCACCTG-3'). When required, 4-MNol was added at a final concentration of $100 \mu \mathrm{M}$. The mixture was incubated for $20 \mathrm{~min}$ on ice followed by $10 \mathrm{~min}$ at room temperature. Complexes were separated on $5 \%$ nondenaturing polyacrylamide gel followed by autoradiography using Phospholmager. 


\section{Western blotting}

HepG2 cells were lysed by incubation on ice for 30 minutes in a buffer containing $50 \mathrm{mM}$ Tris, $\mathrm{pH} 7.5$, $1 \%$ NP40, 0.25\% Na-deoxycholate, $150 \mathrm{mM} \mathrm{NaCl}, 1 \mathrm{mM}$ EDTA, 10\% glycerol and protease inhibitor cocktail (Roche). The lysates were centrifuged at $12000 \mathrm{~g}$ for 15 minutes. The cleared supernatants were separated by $10 \%$ polyacrylamide gel electrophoresis and transferred to nitrocellulose membranes. The membranes were blocked with 5\% BSA-PBS and probed with antibodies against TFIIB (Santa Cruz, sc-225) and COUP-TFII. COUP-TFII antibodies were raised in rabbits using bacterially-expressed recombinant proteins as antigen. When required, cells were treated with $2 \mu \mathrm{M}$ MG132 for 18 hours.

\section{In vitro translation of hCOUP-TFII and limited proteolysis assay}

In vitro translation was performed in reticulocyte lysate using the TNT Promega kit (Promega, France) and $\left[{ }^{35} \mathrm{~S}\right]-$ methionine, in the presence of vehicle (DMSO) or $40 \mu \mathrm{M}$ of 4-MNol. Limited digestion of labeled receptor was carried out with chymotrypsin (SIGMA) at concentrations ranging from 1 to 10 $\mu \mathrm{g} / \mathrm{mL}$. After $10 \mathrm{~min}$ incubation, digestion was stopped with the addition of Laemmli buffer. The samples were boiled $5 \mathrm{~min}$ and separated on a 10\% SDS-PAGE. The radiolabeled products were visualized by autoradiography.

\section{Chromatin immunoprecipitation}

Chromatin immunoprecipitation with HepG2 cells was performed as described in Tatarakis et al. 2008, ${ }^{35}$ using antibodies against acetylated H3 and H4 (Upstate/Millipore), RNA-Polll (Abcam), HNF$4 \alpha$ and COUP-TFII raised in the laboratory. The specificity of the antibodies was verified by IP-western blot assays using crosslinked cellular extracts. Quantitative PCR was performed using primers amplifying the human Cyp7A1 promoter: Sense: 5' GTATTGCAGGTCTCTGATTG 3'; Antisense: 5' CCAGAT CCATTAACTTGAGC 3'.

\section{Structure visualization, binding site prediction and docking analyses}

COUP-TFII (3cjw), RXR $\alpha$ (1xdk) and HNF4 $\alpha$ (1m7w) LBD structures were visualized from pdb files with UCSF Chimera software (version 1.4.1). Q-SiteFinder, Pocket-Finder and PPI-pred on-line softwares (http://www.bioinformatics.leeds.ac.uk) were used with 3cjw pdb file to screen for putative 
ligand binding sites, protein cavities and protein-protein interaction surfaces respectively. ${ }^{18-20}$ Clean hCOUP-TFII (3cjw pdb) input file was generated by using the protein preparation wizard of Gold Suite V5.1 (Cambridge Crystallographic Data Centre, Cambridge). The bond orders were assigned and hydrogen atoms were added. The resulting receptor structure was saved to a mol2 file. The ligand input structures were generated and 3D optimized with MarvinSketch Academic Package (MarvinSketch 5.4.1.1, 2011, ChemAxon http://www.chemaxon.com). The ligand structures were saved as mol2 file. Docking was performed by using chemscore fitness function under "Standard default settings" of GOLD software. Early termination was allowed if 4 solutions were within 1.5 angstroms R.M.S.D.

\section{T3-L1 cell culture and adipocyte differentiation}

3T3-L1 cells were maintained at subconfluence at $37^{\circ} \mathrm{C}$ and $5 \% \mathrm{CO}_{2}$ in proliferation medium (PM): Dulbecco's modified Eagle's medium (DMEM, Gibco) containing high glucose concentration (4.5 g/L), a mix of $100 \mathrm{U} / \mathrm{mL}$ penicilline and $100 \mu \mathrm{g} / \mathrm{mL}$ streptomycine (Gibco), supplemented with $10 \%$ (v/v) calf serum (Cosmic Calf Serum, Hyclone). Before induction of adipocyte differentiation, cells were plated in 12-well plates $\left(50.10^{3}\right.$ cells/well) in PM. When cells reached confluence (day-2, D-2), PM was renewed and cells were maintained 2 days post-confluence until day 0 (D0). At D0, 3T3-L1 cells were induced to differentiate into adipocytes using differentiation medium (DM) using Cosmic Calf Serum into Foetal bovine Serum (Gibco) supplemented with $1 \mu \mathrm{M}$ dexamethasone (Dex). Two days later (day 2, D2), medium was replaced by DM with $1 \mu \mathrm{g} / \mathrm{mL}$ bovine insulin (Ins). Two days later (day 4 , D4), medium was replaced by DM and subsequently renewed every two days until adipocytes reached differentiation (day 8, D8).

\section{Oil Red $O$ staining and quantification of triglycerides}

Eight days after differentiation induction (D8), Oil red $\mathrm{O}(\mathrm{ORO})$ staining and quantification was performed as previously described. ${ }^{36}$ Additionally, intra-cellular triglycerides (TG) were also quantified as described hereafter. Cells were washed with cold PBS and harvested using $200 \mu \mathrm{L}$ of lysis buffer (1X PBS with $1 \%$ SDS). $50 \mu \mathrm{L}$ of lysate was used for protein quantification using the Bio-Rad DC Protein Assay according to the manufacturer's instructions. The remaining lysate was transferred into glass tubes on ice and diluted by adding $600 \mu \mathrm{L}$ of ultra-pure water. Intracellular TG were extracted by 
addition of $3 \mathrm{~mL}$ of chloroform:methanol (2:1). After homogenization and centrifugation at $160 \mathrm{~g}$ for 5 minutes at room temperature, the upper phase and protein disk were discarded. TG were then precipitated by adding $1 \mathrm{~mL}$ of chloroform and Triton-X100 (final concentration 1\%) to the organic phase and samples were dried under nitrogen after homogenization. Pellets were resuspended into 80 $\mu \mathrm{L}$ of ultra-pure water and TG were quantified using the TG PAP-1000 (BioMerieux) according to the manufacturer's instructions and CFAS (Roche Diagnotics) as standard. TG contents were normalized to total protein levels.

\section{Gene expression analysis by RT-PCR and RT-qPCR}

At indicated times, total RNAs were extracted using the Extract-all reagent (Eurobio, Courtabeuf, France) according to the manufacturer's protocol. $500 \mathrm{ng}$ of total RNAs were reverse-transcribed using the High Capacity Reverse Transcription Kit (Applied Biosystem, Life Technologies, Carlsbad, CA, USA) according to the manufacturer's protocol. A 1:20 dilution of cDNA was then amplified by real time qPCR cDNA either in a 7500 Fast real-time PCR system (Applied Biosystems) using TaqMan Gene Expression Assays as recommended by the manufacturer (3T3-L1 cells) or in a CFX96 realtime system (Biorad) using SYBR green (HepG2 cells). Relative gene expression was calculated by the $\Delta \Delta \mathrm{Ct}$ method and final results were expressed as the fold difference relative to control conditions in gene expression normalized to 18S rRNA for 3T3-L1 and RSP28 in HepG2 cells. Primers were provided by Sigma (France) and are listed in Supporting information. Probes used with TaqMan Gene Expression Assays were provided by ThermoFisher Scientific and are listed in Supporting information. For siRNA experiments, HepG2 cells were transfected with scramble or COUP-TFII targeting pools of siRNAs (Trisilencer-27 siRNA duplexes, Origene) as recommended by the manufacturer. After 6 hours of transfection, cells were treated for 48 hours with DMSO or $50 \mu \mathrm{M} 4-\mathrm{MNol}$.

\section{Immunofluorescence}

HepG2 cells $\left(5.10^{5}\right.$ cells per well) were seeded on coverslips in 6 -well plates $24 \mathrm{~h}$ before treatment. Cells were treated two times during $48 \mathrm{~h}$ with $50 \mu \mathrm{M} 4-\mathrm{MNol}$ or $0.5 \%$ DMSO as a negative control. Cells were then washed three times with PBS and fixed with $4 \%$ paraformaldehyde. After three washes in PBS, unspecific sites were saturated using blocking buffer (PBS with $0.1 \%$ triton, $5 \%$ foetal bovine serum 5 \%). Then, cells were incubated with primary antibody (anti-COUP-TFII, 1/1000 in 
blocking buffer) during $16 \mathrm{~h}$ at $4{ }^{\circ} \mathrm{C}$. Cells were next incubated with secondary antibody (anti-rabbit antibody coupled to Alexafluor) during $2 \mathrm{~h}$ at room temperature. Following three washes in PBS, coverslips were mounted on glass slides with DAPI (Vectashield). Images were acquired on an Apotome microscope (Zeiss). For each condition, 14 microscopic fields with an average of 200 cells per field were analysed for the presence of 1 or several fluorescent foci in the nucleus or in the cytosol.

\section{Acknowledgments}

This work was funded by the European Community (QLG1-CT-2001-01513), the Centre National pour la Recherche Scientifique and the Ministère de l'Enseignement Supérieur et de la Recherche. This work was also supported by grants from "European Genomic Institute for Diabetes" (E.G.I.D., ANR-10LABX-46). F.O. and C.G. were supported by OSEO-ANVAR (IT-DIAB). B.S. is a member of the Institut Universitaire de France.

\section{References}

1. Germain, P., Staels, B., Dacquet, C., Spedding, M., and Laudet, V. (2006) Overview of nomenclature of nuclear receptors, Pharmacol. Rev. 58, 685-704.

2. Adam, F., Sourisseau, T., Métivier, R., Le Page, Y., Desbois, C., Michel, D., and Salbert, G. (2000) COUP-TFI (chicken ovalbumin upstream promoter-transcription factor I) regulates cell migration and axogenesis in differentiating P19 embryonal carcinoma cells, Mol. Endocrinol. 14, 1918-1933.

3. Armentano, M., Chou, S.J., Tomassy, G.S., Leingärtner, A., O'Leary, D.D., and Studer, M. (2007) COUP-TFI regulates the balance of cortical patterning between frontal/motor and sensory areas, Nat. Neurosci. 10, 1277-1286.

4. Pereira, F.A., Tsai, M.J., and Tsai, S.Y. (2000) COUP-TF orphan nuclear receptors in development and differentiation, Cell. Mol. Life Sci. 57, 1388-1398.

5. Kurihara, I., Lee, D.K., Petit, F.G., Jeong, J., Lee, K., Lydon, J.P., DeMayo, F.J., Tsai, M.J., and Tsai, S.Y. (2007) COUPTFII mediates progesterone regulation of uterine implantation by controlling ER activity, PLoS Genet. 3(6), e102. 
6. Naka, H., Nakamura, S., Shimazaki, T., and Okano, H. (2008) Requirement for COUP-TFI and COUP-TFII in the temporal specification of neural stem cells in CNS development, Nat. Neurosci. 11, 1014-1023.

7. Xu, Z., Yu, S., Hsu, C.H., Eguchi, J., and Rosen, E.D. (2008) The orphan nuclear receptor chicken ovalbumin upstream promoter-transcription factor II is a critical regulator of adipogenesis, Proc. Natl. Acad. Sci. U.S.A. 105, 2421-2426.

8. Kruse, S.W., Suino-Powell, K., Zhou, X.E., Kretschman, J.E., Reynolds, R., Vonrhein, C., Xu, Y., Wang, L., Tsai, S.Y., Tsai, M.J., and Xu, H.E. (2008) Identification of COUP-TFII orphan nuclear receptor as a retinoic acid activated receptor, PLoS Biol. 6(9), e227.

9. Flaig, R., Greschik, H., Peluso-Iltis, C., and Moras, D. (2005) Structural basis for the cell-specific activities of the NGFI-B and the Nurr1 ligand-binding domain, J. Biol. Chem. 280, 19250-19258.

10. Wang, Z., Benoit, G., Liu, J., Prasad, S., Aarnisalo, P., Liu, X., Xu, H., Walker, N.P., and Perlmann, T. (2003) Structure and function of Nurr1 identifies a class of ligand-independent nuclear receptors, Nature 423, 555-560.

11. Kallen, J., Schlaeppi, J.M., Bitsch, F., Filipuzzi, I., Schilb, A., Riou, V., Graham, A., Strauss, A., Geiser, M., and Fournier, B. (2004) Evidence for ligand-independent transcriptional activation of the human estrogen related receptor alpha (ERRalpha): crystal structure of ERRalpha ligand binding domain in complex with peroxisome proliferator-activated receptor coactivator-1alpha, J. Biol. Chem. $279,49330-49337$.

12. Li, Y., Choi, M., Cavey, G., Daugherty, J., Suino, K., Kovach, A., Bingham, N.C., Kliewer, S.A., and Xu, H.E. (2005) Crystallographic identification and functional characterization of phospholipids as ligands for the orphan nuclear receptor steroidogenic factor-1, Mol. Cell 17, 491-502.

13. Hintermann, S., Chiesi, M., von Krosigk, U., Mathé, D., Felber, R., and Hengerer, B. (2007) Identification of a series of highly potent activators of the Nurr1 signaling pathway, Bioorg. Med. Chem. Lett. 17, 193-196.

14. Poppe, L., Harvey, T.S., Mohr, C., Zondlo, J., Tegley, C.M., Nuanmanee, O., and Cheetham, J. (2007) Discovery of ligands for Nurr1 by combined use of NMR screening with different isotopic and spin-labeling strategies, J. Biomol. Screen. 12, 301-311. 
15. Greschik, H., Flaig, R., Renaud, J.P., and Moras, D. (2004) Structural basis for the deactivation of the estrogen related receptor gamma by diethylstilbestrol or 4-hydroxytamoxifen and determinants of selectivity, J. Biol. Chem. 279, 33639-33646.

16. Lipfert, L., Fisher, J.E., Wei, N., Scafonas, A., Su, Q., Yudkovitz, J., Chen, F., Warrier, S., Birzin, E.T., Kim, S., Chen, H.Y., Tan, Q., Schmidt, A., Dininno, F., Rohrer, S.P., Hammond, M.L., Rodan, G.A., Freedman, L.P., and Reszka, A.A. (2006) Antagonist-induced, activation function-2-independent estrogen receptor alpha phosphorylation, Mol. Endocrinol. 20, 516-533.

17. Long, X., and Nephew, K.P. (2006) Fulvestrant (ICl 182,780)-dependent interacting proteins mediate immobilization and degradation of estrogen receptor-alpha, J. Biol. Chem. 281, 9607-9615

18. Laurie, A.T., and Jackson, R.M. (2005) Q-SiteFinder: an energy-based method for the prediction of protein ligand binding sites, Bioinformatics 21, 1908-1916.

19. Hendlich, M., Rippmann, F., and Barnickel, G. (1997) LIGSITE: automatic and efficient detection of potential small molecule-binding sites in proteins, J. Mol. Graph. Model. 15, 359-363.

20. Bradford, J.R., and Westhead, D.R. (2005) Improved prediction of protein-protein binding sites using a support vector machines approach, Bioinformatics 21, 1487-1494.

21. Sun, A., Moore, T.W., Gunther, J.R., Kim, M.-S., Rhoden, E., Du, Y., Fu, H., Snyder, J.P., and Katzenellenbogen, J.A. (2011) Discovering small molecule estrogen receptor $\alpha /$ coactivator binding inhibitors, and models for enhanced potency, ChemMedChem 6, 654-666.

22. Benkoussa, M., Nominé, B., Mouchon, A., Lefebvre, B., Bernardon, J.M., Formstecher, P., and Lefebvre, P. (1997) Limited proteolysis for assaying ligand binding affinities of nuclear receptors, Recept. Signal. Transduct. 7, 257-267.

23. Shoji, O., Wiese, C., Fujishiro, T., Shirataki, C., Wunsch, B., and Watanabe, Y. (2010) Aromatic C$\mathrm{H}$ bond hydroxylation by P450 peroxygenases: a facile colorimetric assay for monooxygenation activities of enzymes based on Russig's blue formation, J. Biol. Inorq. Chem. 15, 1109-1115.

24. Zhang, P., Bennoun, M., Gogard, C., Bossard, P., Leclerc, I., Kahn, A., and Vasseur-Cognet, M. (2002). Expression of COUP-TFII in metabolic tissues during development, Mech. Dev. 119,109-114.

25. Ladias, J.A., Hadzopoulou-Cladaras, M., Kardassis, D., Cardot, P., Cheng, J., Zannis, V., and Cladaras, C. (1992). Transcriptional regulation of human apolipoprotein genes ApoB, ApoCIII, and ApoAll by members of the steroid hormone receptor superfamily HNF-4, ARP-1, EAR-2, and EAR-3, J. Biol. Chem. 267, 15849-15860. 
26. Mietus-Snyder, M., Sladek, F.M., Ginsburg, G.S., Kuo, C.F., Ladias, J.A., Darnell, J.E.Jr, and Karathanasis, S.K. (1992) Antagonism between apolipoprotein Al regulatory protein 1, Ear3/COUP$\mathrm{TF}$, and hepatocyte nuclear factor 4 modulates apolipoprotein CIII gene expression in liver and intestinal cells, Mol. Cell. Biol. 12, 1708-1718.

27. Ochoa, A., Bovard-Houppermans, S., and Zakin, M.M. (1993) Human apolipoprotein A-IV gene expression is modulated by members of the nuclear hormone receptor superfamily, Biochem. Biophys. Acta 1210, 41-47.

28. Crestani, M., Sadeghpour, A., Stroup, D., Galli, G., and Chiang, J.Y. (1998) Transcriptional activation of the cholesterol 7alpha-hydroxylase gene (CYP7A) by nuclear hormone receptors, J. Lipid Res. 39, 2192-2200.

29. Kang, S., Spann, N.J., Hui, T.Y., and Davis, R.A. (2003) ARP-1/COUP-TF II determines hepatoma phenotype by acting as both a transcriptional repressor of microsomal triglyceride transfer protein and an inducer of CYP7A1, J. Biol. Chem. 278, 30478-30486.

30. Stroup, D., and Chiang, J.Y. (2000) HNF4 and COUP-TFII interact to modulate transcription of the cholesterol 7alpha-hydroxylase gene (CYP7A1), J. Lipid Res. 41, 1-11.

31. Okamura. M.. Kudo, H., Wakabayashi, K., Tanaka, T., Nonaka, A., Uchida, A., Tsutsumi, S., Sakakibara, I., Naito, M., Osborne, T.F., Hamakubo, T., Ito, S., Aburatani, H., Yanagisawa, M., Kodama, T., and Sakai, J. (2009) COUP-TFII acts downstream of Wnt/beta-catenin signal to silence PPARgamma gene expression and repress adipogenesis, Proc. Natl. Acad. Sci. U.S.A. 106, 58195824.

32. Li, L., Xie, X., Qin, J., Jeha, G.S., Saha, P.K., Yan, J., Haueter, C.M., Chan, L., Tsai, S.Y., and Tsai, M.J. (2009) The nuclear orphan receptor COUP-TFII plays an essential role in adipogenesis, glucose homeostasis, and energy metabolism, Cell Metab. 9, 77-87.

33. Le Guével, R., Oger, F., Lecorgne, A., Dudasova, Z., Chevance, S., Bondon, A., Barath, P., Simonneaux, G., and Salbert, G. (2009) Identification of small molecule regulators of the nuclear receptor HNF4alpha based on naphthofuran scaffolds, Bioorg. Med. Chem. 17, 7021-7030.

34. Le Guével, R., and Pakdel, F. (2001) Streamlined beta-galactosidase assay for analysis of recombinant yeast response to estrogens, Biotechniques 30, 1000-1004. 
35. Tatarakis, A., Margaritis, T., Martinez-Jimenez, C.P., Kouskouti, A., Mohan, W.S. 2nd, Haroniti, A., Kafetzopoulos, D., Tora, L., and Talianidis, I. (2008) Dominant and redundant functions of TFIID involved in the regulation of hepatic genes, Mol. Cell 31, 531-543.

36. Dubois-Chevalier, J., Oger, F., Dehondt, H., Firmin, F.F., Gheeraert, C., Staels, B., Lefebvre, P. and Eeckhoute, J. (2014) A dynamic CTCF chromatin binding landscape promotes DNA hydroxymethylation and transcriptional induction of adipocyte differentiation, Nucleic Acids Res. 42, 10943-10959.

\section{Figure legends}

Figure 1: Definition of a putative ligand binding site in COUP-TFII LBD and mutational analysis in yeast one-hybrid system. (A) Hydrophobicity surface view of COUP-TFII LBD (3cjw). Color code varies from blue for hydrophilic residues to red for hydrophobic residues. The position of a small cavity therefater called "surface pocket" is indicated by an arrow. (B) Sequence of the LBD protein crystallized in 3cjw. Numbering on the left refered to the crystallized protein. Positions of the first and last residues with respect to the full-length COUP-TFII protein are indicated. Residues lining the predicted ligand binding site are highlighted in color (red for polar residues and green for non-polar residues). These residues are mainly included in helices $\alpha 3, \alpha 7$ and $\alpha 10 / 11$. (C) Close-up view of the surface pocket. (D) List of the mutated residues analyzed in yeat one-hybrid assay. Their inclusion in the filled classical ligand binding pocket or in the surface pocket is indicated. (E and F) One-hybrid assay of wt and mutated COUP-TFII. Bars indicate $\beta$-galactosidase activity relative to wt COUP-TFII. Results are shown as means $+/$ - SEM $(n=3)$.

Figure 2: Naphthol derivatives inhibit COUP-TFII transcriptional activity. (A) Dose-effect of naphthol derivatives in yeast one-hybrid assay. Beta-galactosidase activities are shown relative to untreated yeast cells as means + - SEM $(n=3)$. (B) Structures of two active compounds. (C) Docking of 4-MNol in the surface pocket. The pocket is shown as hydrophobicity surface (blue: hydrophilic, red: hydrophobic). 4-MNol atoms are colored as follow: carbon: white, hhydrogen: white, oxygen: red, lone pairs of electrons: magenta. (D) Residues from the surface pocket predicted to contact 4-MNol. Pseudobonds were determined with Chimera and are shown as green lines. Hydophobic contacts are 
detected for 1212, A287, 1292, L381 and F382, whereas lone pairs of electrons could establish hydrogen bonds with $\mathrm{C} 213$ and R385. Color code for 4-MNol atoms is identical to (C) except for carbon atoms which are colored in blue. (E) C213A COUP-TFII mutant is less sensitive to 4-MNol inhibition. Dose-response curves of $\beta$-galactosidase activities obtained with wt or C213A COUP-TFII in yeast one-hybrid assays. Data are shown as in (A). (F) Limited proteolysis assay of wtCOUP-TFII, in vitro tanslated in the presence or not of $100 \mu \mathrm{M}$ 4-MNol and incubated with increasing concentrations of chymotrypsin (black triangles). The full-length protein is indicated by an arrow and a vertical bar indicates the degradation products resistant to protease.

Figure 3: Altered binding of COUP-TFII to DNA in the presence of 4-MNol. (A) 4-MNol does not inhibit in vitro translation of wtCOUP-TFII. Translation was run in the presence of ${ }^{35} \mathrm{~S}$-methionine in the presence or not of $100 \mu \mathrm{M} 4-\mathrm{MNol}$, and proteins were separated by SDS-PAGE before autoradiography. The positions of molecular mass markers are indicated on the left. (B) EMSA of wtCOUP-TFII in vitro translated in the presence of $100 \mu \mathrm{M}$ 4-MNol with a radio-labeled DR1 probe. Unprogrammed lysate (UPL) was used as a control. The free probe is indicated by a black arrowhead, a non-specific shifted band by a white arrowhead, and the COUP-TFII/DR1 probe complex by an asterisk. (C and D) EMSA of wt (C) and C213A (D) COUP-TFIl binding to the DR1 probe in two different conditions: (1) COUP-TF was in vitro translated in the presence of $100 \mu \mathrm{M}$ 4-MNol or vehicle (ethanol) and (2) COUP-TFII, in vitro translated in the presence of ethanol, was incubated with increasing concentrations of 4-MNol before addition of the probe, whereas COUP-TFII translated in the presence of 4-MNol was incubated with increasing concentrations of DTT before addition of the probe. Black arrowheads indicate position of the free probe.

Figure 4: 4-MNol reduces expression of the COUP-TFIl target gene Cyp7a1. (A) Toxicity assay of 4-MNol. HepG2 cells were treated for $48 \mathrm{~h}$ with DMSO or increasing concentrations of 4-MNol. Cell viability was determined by trypan blue exclusion. (B) RT-qPCR analysis of ApoA1 and Cyp7a1 mRNA levels. Data were expressed as $\Delta \Delta$ ct with RSP28 as a reference. Results are expressed as \% of expression of the corresponding genes in cells treated with DMSO (mean +/- SEM, $n=9$ ). (C) RTqPCR analysis of COUP-TFII Cyp7a1 and ApoA1 mRNA levels in cells treated with DMSO (black bars) or $50 \mu \mathrm{M}$ 4-MNol (grey bars) for 48 hours after transfection of either scramble siRNA or siRNAs 
targeting COUP-TFII (mean +/- SEM, $n=6$ ). Statistical significance was determined using unpaired $t-$ test. (D) ChIP assay of the indicated proteins and histone marks at the Cyp7a1 promoter in HepG2 cells treated for 2 days with DMSO (blacks bars) or $25 \mu \mathrm{M}$ 4-MNol (grey bars). The scheme depicts the amplified region (primers figured as arrows) which includes the bile acid reponse element (BARE) known to bind HNF4 $\alpha$ and the TATA box. Statistical significance was determined using unpaired t-test $\left({ }^{* *} p<0.01,{ }^{* * *} p<0.001\right)$. (E) Western blot analysis of COUP-TFII and TFIIB in HepG2 cells treated either with 4-MNol or the proteasome inhibitor MG132 or both. (F) Immunofluorescence analysis of COUP-TFII distribution in HepG2 cells treated or not with $50 \mu \mathrm{M}$ of 4-MNol. Cells either presented a homogeneous nuclear distribution of COUP-TFII or a punctuated distribution with the different phenotypes examplified in the pictures: 1 , a single foci per nucleus; 2 , several foci per nucleus; 3 , several foci in the cytosol. The occurrence of each phenotype was determined and data are presented as the ratio of the occurrence in 4-MNol- versus DMSO-treated cells (mean +/- SEM, $n=13$ ).

Figure 5: 4-MNol enhances conversion of 3T3-L1 fibroblasts to adipocytes in vitro. (A) Schematic representation of the protocol used to induce partial differentiation of 3T3-L1 cells in adipocytes. (B) Toxicity assay of 4-MNol. 3T3-L1 cells were treated with DMSO or increasing concentrations of 4-MNol as described in (A). Cell viability was determined by trypan blue exclusion. (C) ORO-staining of 3T3-L1 cells 8 days post-induction of differentiation [upper: macroscopic view; bottom: microscopic view (x20)]. (D) Quantification of ORO staining (mean +/- SEM, $n=3$ ). (E) Quantification of TG content of 3T3-L1 -derived adipocytes (mean +/- SEM, $n=3$ ). (F) RT-qPCR analysis of PPARy and Adiponectin mRNA levels. Results are displayed relatively to the mRNA expression levels of PPARY and adiponectin at each day post-induction of partial adipocyte differentiation without 4-MNol (set to 1). Histograms represent means \pm SEM of a representative experiment performed in triplicates. Statistical significance was determined using unpaired t-test (* $\left.p<0.05,{ }^{* *} p<0.01\right)$. 
A

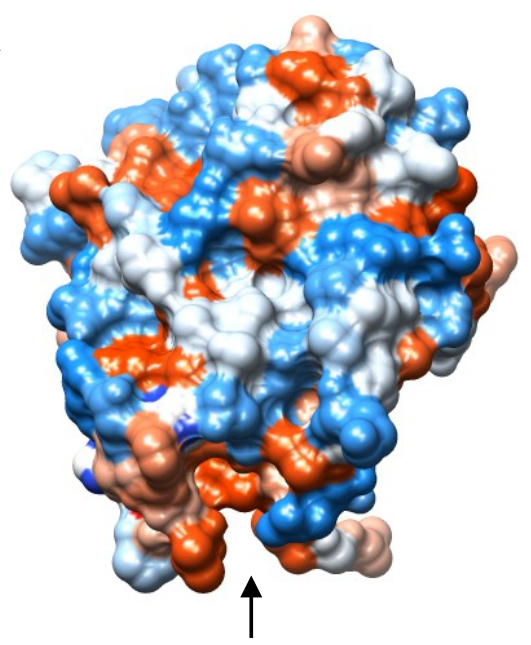

C

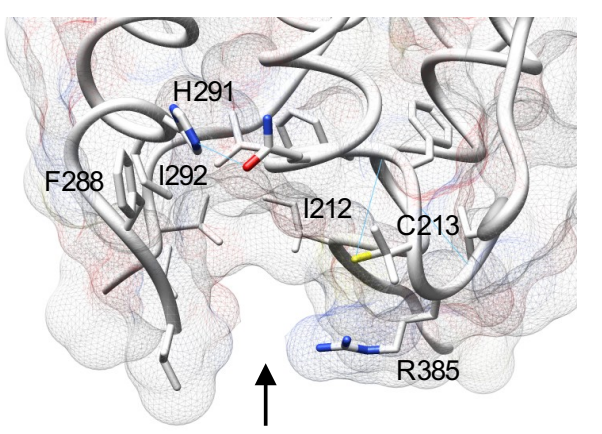

B

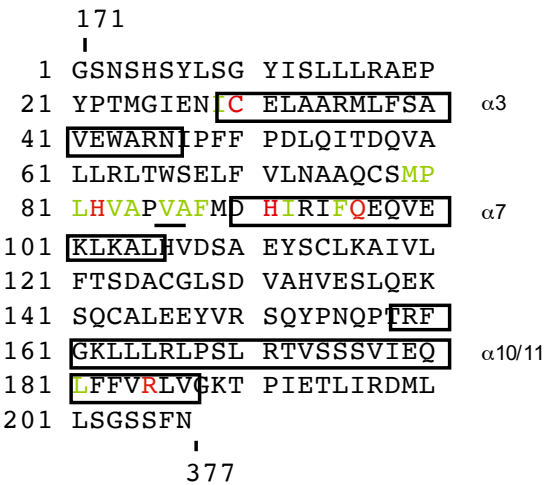

D

\begin{tabular}{|lcc|}
\hline Residue & $\begin{array}{c}\text { Filling classical } \\
\text { pocket }\end{array}$ & $\begin{array}{c}\text { In surface } \\
\text { cavity }\end{array}$ \\
\hline I212 & & X \\
C213 & & X \\
W249 & $X$ & \\
F253 & $X$ & $X$ \\
F288 & & $X$ \\
H291 & & \\
F295 & $X$ & \\
\hline
\end{tabular}

E

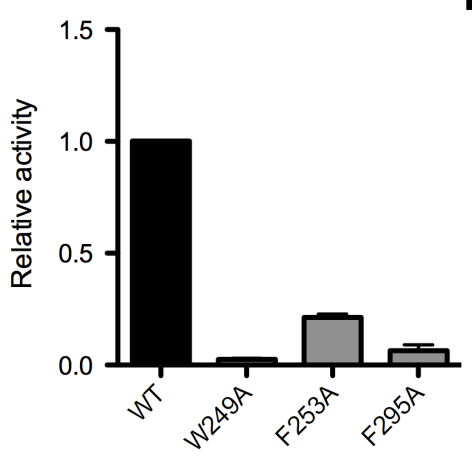

$\mathbf{F}$

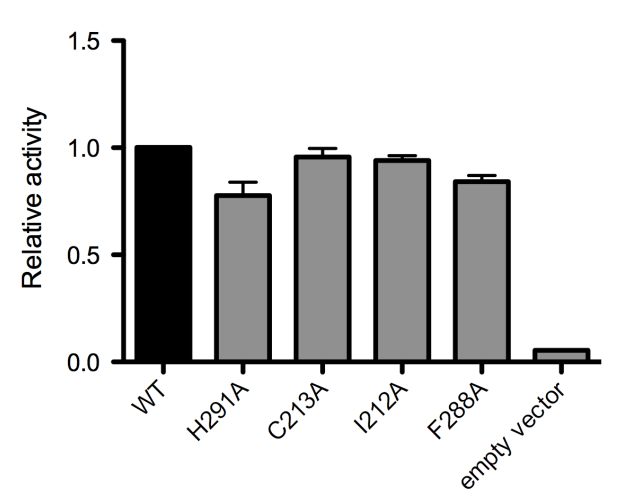

Fig. 1, Le Guével et al. 
A

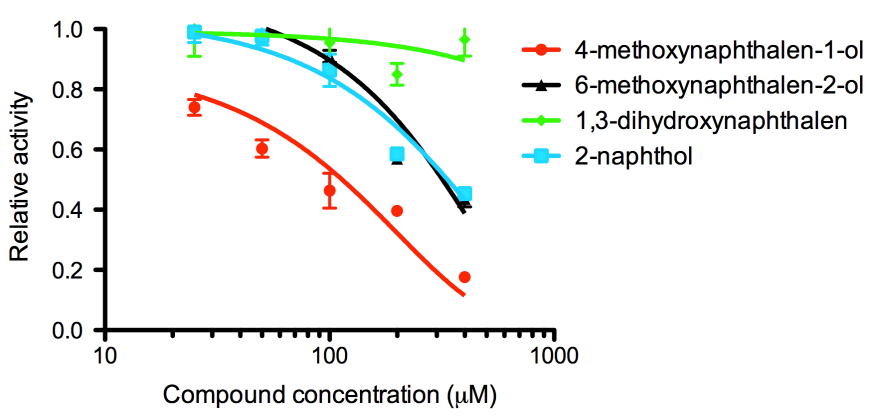

B<smiles>COc1ccc(O)c2ccccc12</smiles>

4-methoxynaphthal en e-1-ol

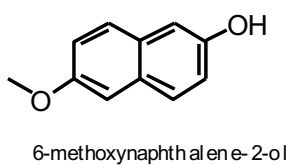

C

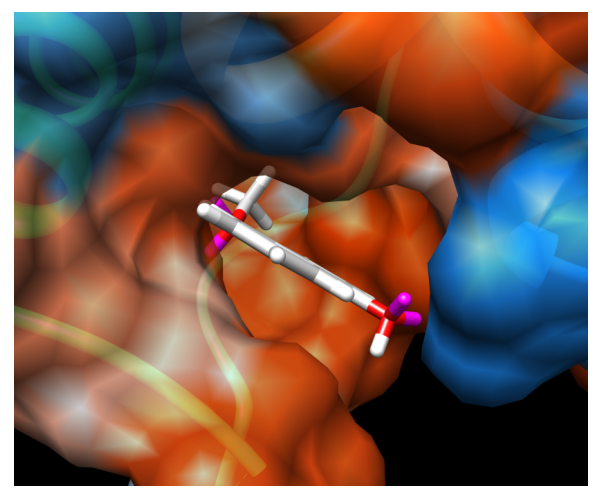

E

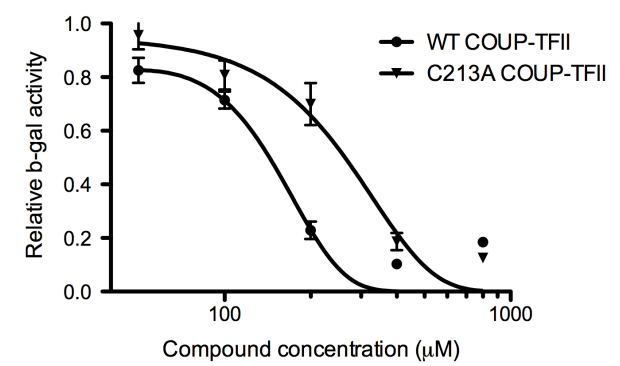

D

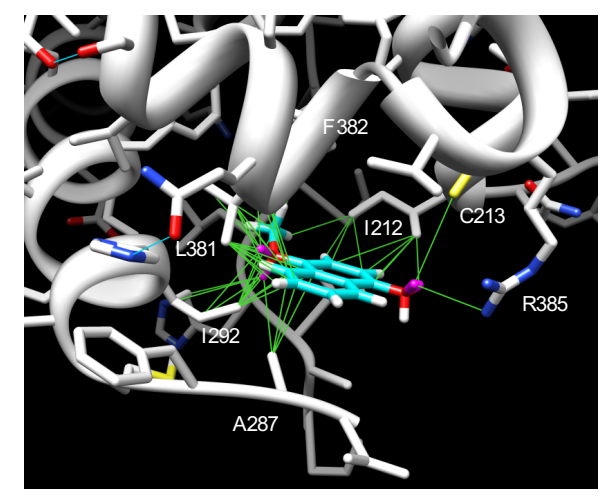

$\mathbf{F}$

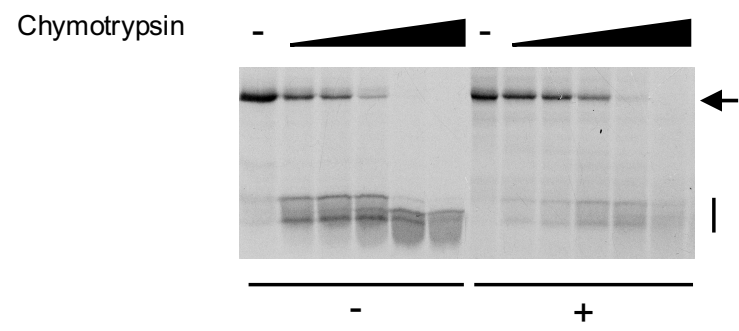

4-methoxynaphthalen-1-ol

Fig. 2, Le Guével et al. 


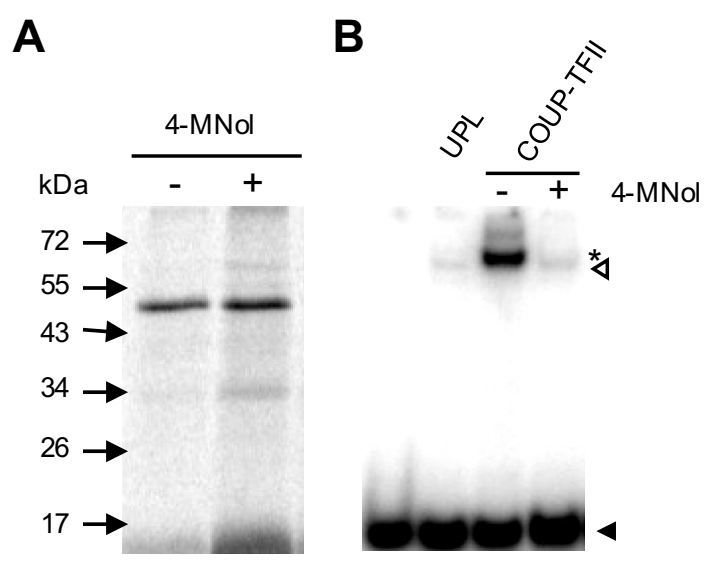

C

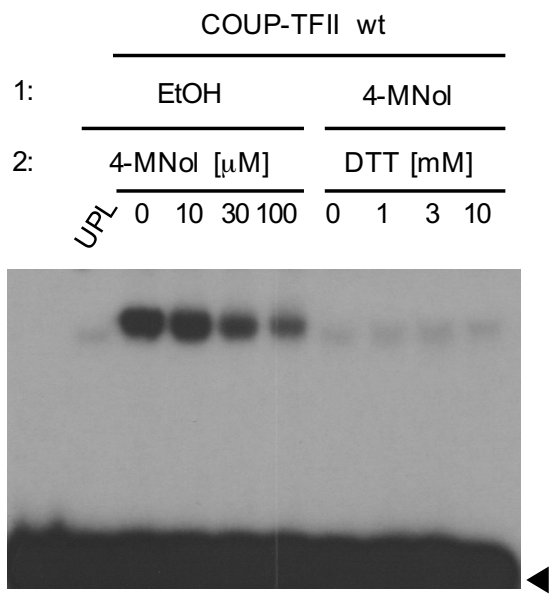

1: Present in TnT reaction

2: Added to EMSA reaction

D

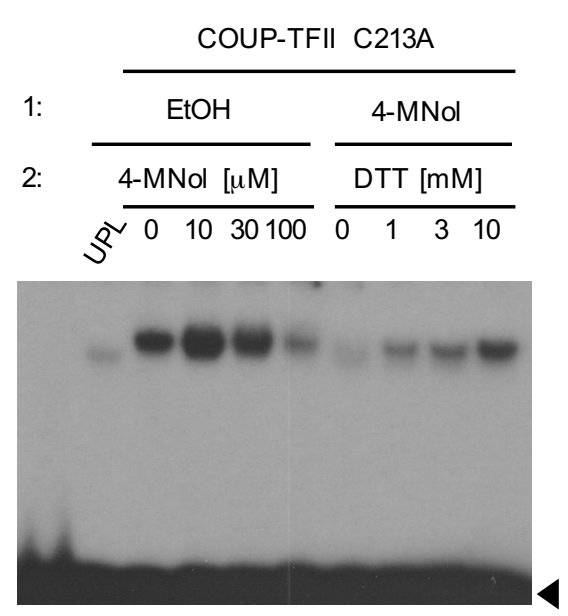

1: Present in TnT reaction

2: Added to EMSA reaction

Fig. 3, Le Guével et al. 
A

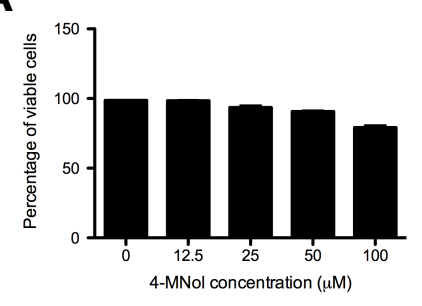

C

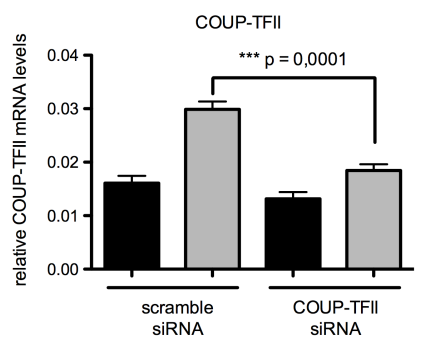

D
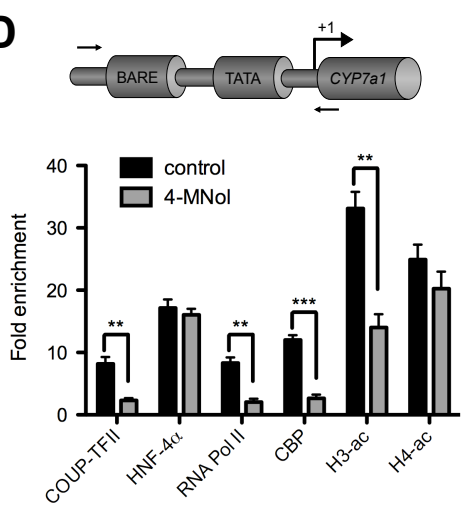

B
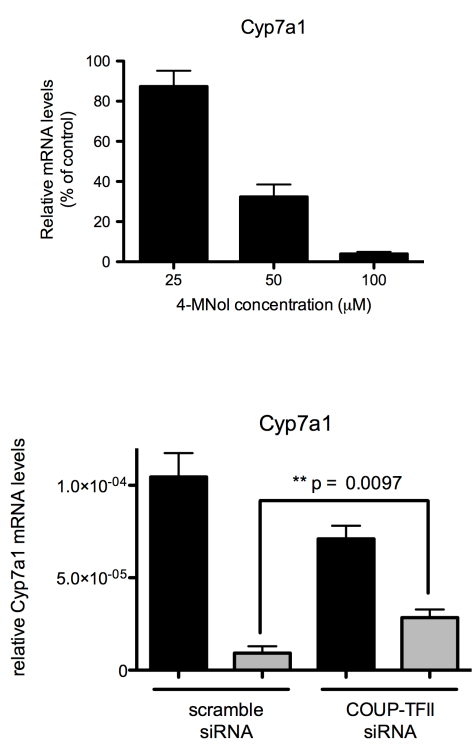

E

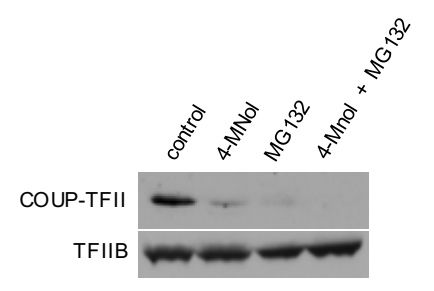

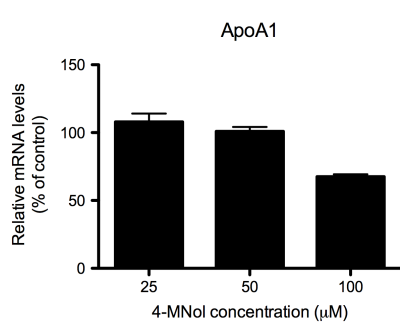

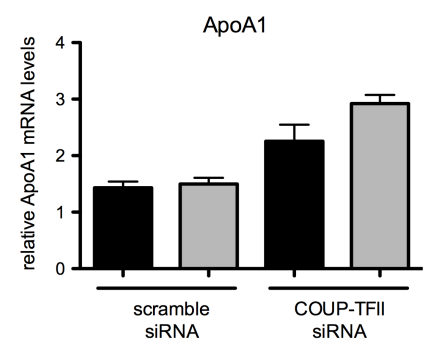

$\mathbf{F}$
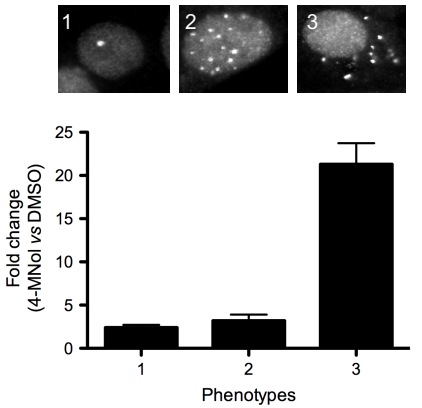

Fig. 4, Le Guével et al. 
A

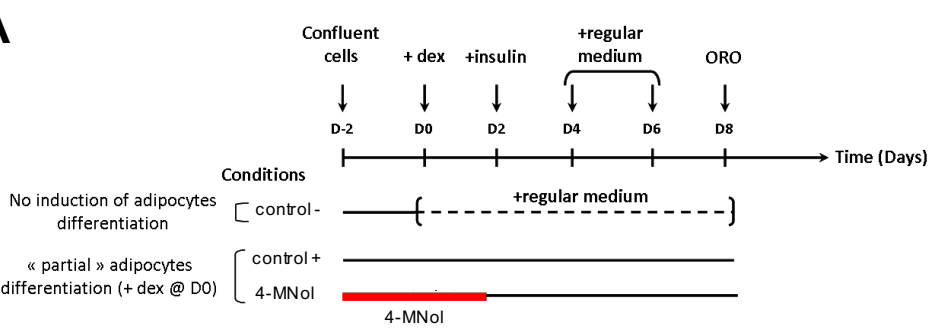

B

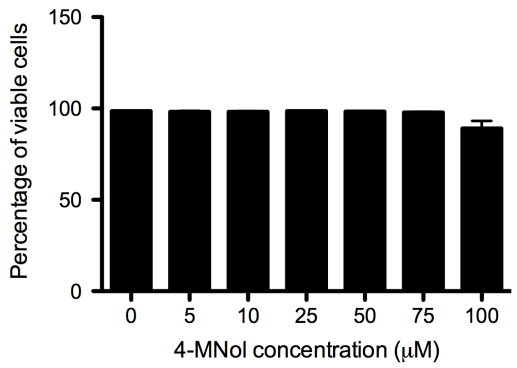

C

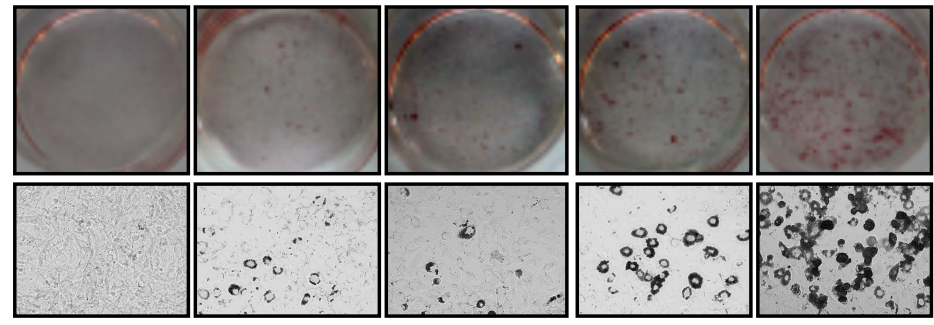

Adipocyte differentiation

$50 \mu \mathrm{M}$

$75 \mu \mathrm{M}$

$100 \mu \mathrm{M}$

D

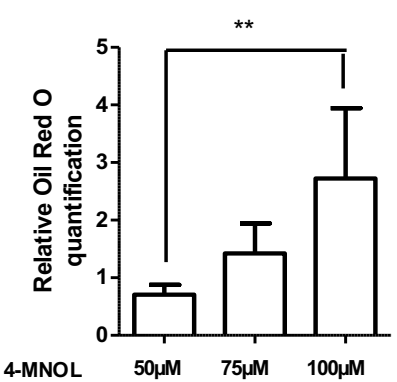

E

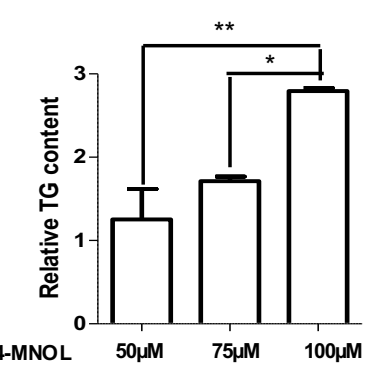

$\mathbf{F}$
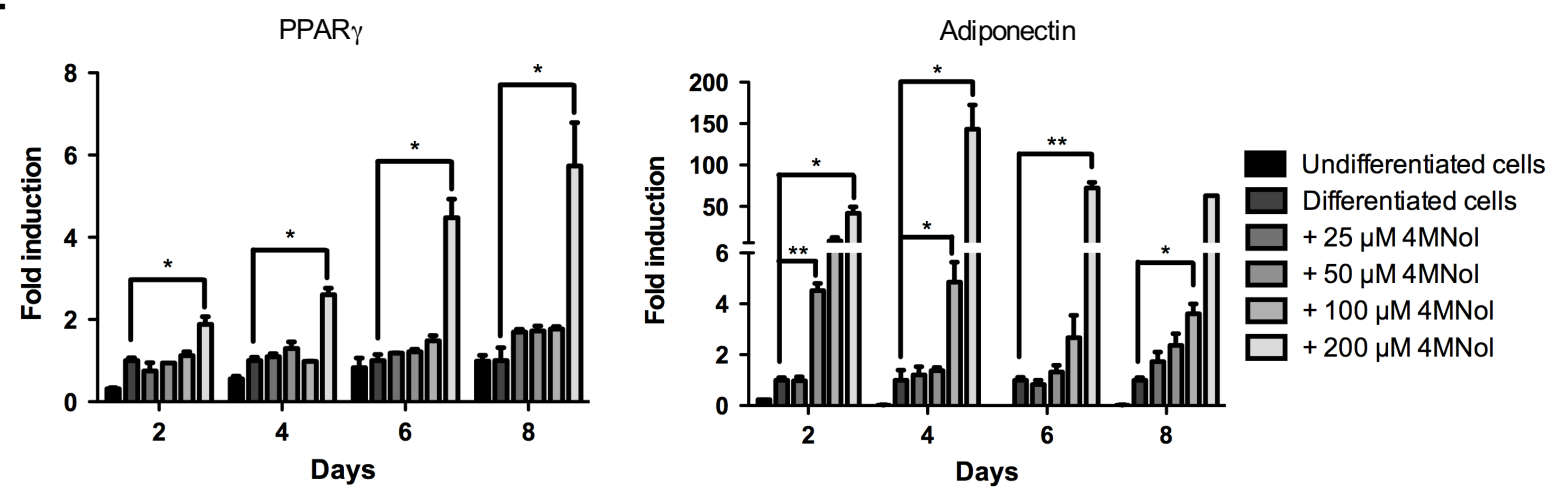

Fig. 5, Le Guével et al. 


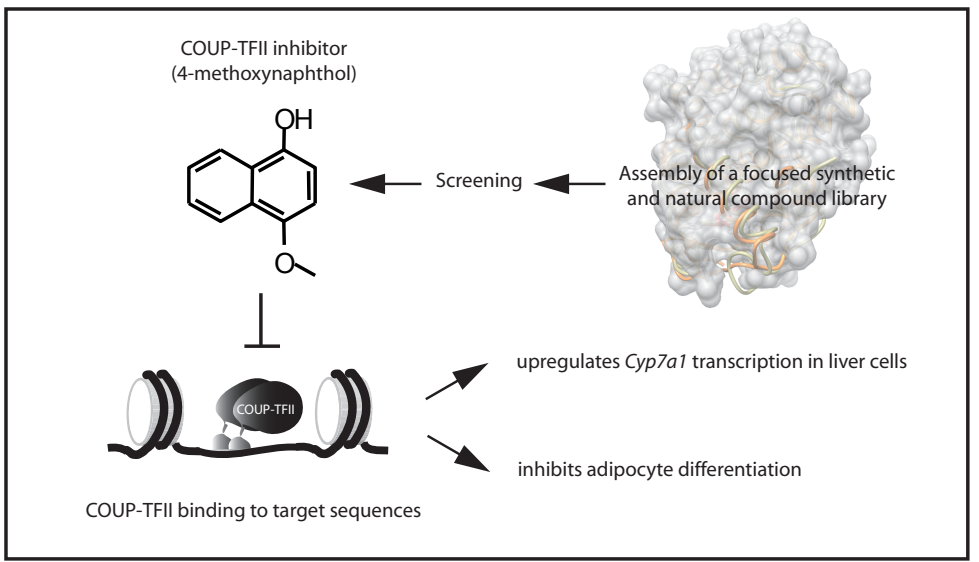

